\title{
Analysis of the Thermo-Mechanical Behavior of Composite Materials based on Plasticized Cassava Starch Reinforced with Coconut Fibers
}

\author{
Ahmed Doumbia* and Pierre J. M. R. Dable \\ Department of Mechanics and Energy, National Polytechnic Institute Felix Houphouet-Boigny of Yamoussoukro, \\ IvoryCoast; fackoly1@gmail.com, pjmardable@yahoo.com
}

\begin{abstract}
Objective: In our previous work, we have developed and characterized a composite material based on cassava starch reinforced with coconut fibers. We will modelyse and simulate its thermo-mechanical behavior. Methodology: For this, we start from Hooke's law to establish a behavior model to evaluate the Young's modulus of each phase (matrix and fibers) as a function of temperature. This is then used in different homogenization models (Voigt and Mori-Tanaka) to approximate the thermoelastic behavior of the composite. Finding: It is noted that the mechanical behavior of the material deteriorates when the temperature increases. Applications: The type of behavior obtained is compared with other previous general results on materials. The biodegradable composite obtained can be used in the field of plastics in the concept of sustainable development.
\end{abstract}

Keywords: Cassava Starch, Coconut Fibers, Composite Materials,Thermo-Mechanical Behavior, Young Modulus

\section{Introduction}

This manuscript is part of the work in which we produced our previous study ${ }^{1}$. It was a question of elaborating and characterizing a composite material based on cassava starch reinforced by coconut fibers. Several tests were carried out. The results showed that the temperature has an effect on the structure of the composite. This affects its mechanical properties. In this study, we establish a method for predicting the elastic behavior of the material as a function of temperature. The composite being bi phasic (matrix + reinforcement), the volume fraction of each phase has an influence on the resistance to a mechanical load of the material. We will also simulate the effect of the variation of the volume contribution of each constituent on the behavior. A model of behavior conceived from the
Hooke law makes it possible to determine the modulus of elasticity of each phase as a function of the temperature. The homogenization models of Mori-Tanaka ${ }^{2}$ and Voigt ${ }^{\underline{3}}$ will allow approximating the modulus of elasticity of the composite.

\section{Law of Thermoelastic Behavior}

Since the material is homogeneous isotropic $\frac{1}{\text {, }}$ if it is submitted to both mechanical and thermal stress, the resulting stress is written:

$$
\sigma=\sigma^{m e ́ c a}+\sigma^{t h e r}
$$

With $\sigma^{\text {méca }}(\mathrm{GPa})$ the mechanical stress 
And $\sigma^{\text {ther }}(\mathrm{GPa})$ the thermal stress.

On the other hand, Hooke's law $w^{4}$ gives the relation between the mechanical stress $\sigma^{\text {méca }}(\mathrm{GPa})$ and the deformation $\varepsilon$ in the domain of elastic behavior:

$$
\sigma^{m e ́ c a}=E \varepsilon
$$

Where $\mathrm{E}(\mathrm{GPa})$ is the modulus of elasticity or Young's modulus of the material.

Taking into account (1) and (2), we have:

$$
\sigma-\sigma^{\text {ther }}=E \varepsilon
$$

From where:

$\varepsilon=\frac{\sigma-\sigma^{\text {ther }}}{E}$

By definition [4]:

$$
\sigma^{\text {ther }}=E_{0} \alpha_{\text {ther }} \Delta T
$$

With $\Delta T=T-T_{0}$

Where $\mathrm{T}_{0}\left({ }^{\circ} \mathrm{C}\right)$ is the initial temperature (ordinary), $\mathrm{T}$ $\left({ }^{\circ} \mathrm{C}\right)$ is the temperature at the current moment, $\mathrm{E}_{0}(\mathrm{GPa})$ is the modulus of elasticity at temperature and $\mathrm{T}_{0}\left(/{ }^{\circ} \mathrm{C}\right)$ is the coefficient of thermal expansion of the material $\alpha_{\text {ther }}$.

In our previous publication, the tensile curve shows that the material adopts an elastic behavior as long as the stress is lower than a maximum value $\sigma_{e}(\mathrm{GPa})$ (called resistance at the elastic limit or elastic limit $R e$ ) determined experimentally $\underline{1-5}$. The greatest stress that the material can withstand while remaining elastic is this value $^{\frac{1-4}{4}}$. At the limit, we must have:

$$
\sigma=R_{p e}
$$

With:

$$
R_{p e}=\frac{\mathrm{R}_{\mathrm{e}}}{\mathrm{C}_{\mathrm{s}}}
$$

Where $R_{p e}(G P a)$ is the practical resistance to extension and $\mathrm{C}_{\mathrm{s}}$ is the factor of security.

Taking into account (4), (5) and (6), it follows that:

$$
\varepsilon=\frac{1}{C_{s} E}\left(R_{e}-C_{s} E_{0} \alpha_{t h e r} \Delta T\right)
$$

Where:

$$
E=\frac{1}{C_{s} \varepsilon}\left(R_{e}-C_{s} E_{0} \alpha_{\text {ther }} \Delta T\right)
$$

Assuming the following initial conditions:

$$
\begin{aligned}
& \text { at } T_{0}: E=E_{0} \\
& \text { and } \varepsilon^{\text {ther }}=\alpha_{\text {ther }}\left(T_{0}-T_{0}\right)=0
\end{aligned}
$$

deformation)

We obtain :

$$
\varepsilon=\frac{R_{e}}{C_{s} E_{0}}
$$

Finally, (9) and (10) give:

$$
E=E_{0} \frac{R_{e}-C_{s} E_{0} \alpha_{t h e r} \Delta T}{R_{e}}
$$


Equation (11) allows determining the modulus of elasticity of each phase of the composite as a function of temperature.

\section{Homogenization Models}

\subsection{Methodology}

The purpose of the homogenization process is to predict the mechanical behavior of the multiphase material by using the information related to the microstructure. The composite is composed of a matrix, denoted by the index " $M$ " and inclusions (reinforcements) denoted by the index " $r$ ". The expression of stiffness tensor $\mathrm{C}$ of the material is given by

$$
C=\sum_{r=0}^{n} f^{r} C^{r} A^{r}
$$

Where $\mathrm{f}^{\mathrm{r}}$ is the volume fraction of the phase $\mathrm{r}, \mathrm{C}^{\mathrm{r}}$ is the average stiffness tensor of $r, n$ is the number of subfamilies of inclusions and $\mathrm{A}^{\mathrm{r}}$ is called the deformation localization tensor.

The knowledge $\mathrm{A}^{\mathrm{r}}$ of is sufficient to determine the following approximations:

\subsection{Mori-Tanaka Model (MT)}

This approximation considers a volume fraction $F^{r}$ of inclusions of the same shape and orientation in the matrix. The volume fraction $F^{r}$ of inclusions can be subdivided into n sub-families $f^{r}$ with different constants of elasticity $C^{r}$ as:

$$
\sum_{r=1}^{n} f^{r}=F^{r}
$$

The principle of approximation of the effective modulus of elasticity of the material by this model consists in considering, for each phase, an equivalent unique inclusion, in the supposed infinite matrix phase, by supposing that this inclusion is subjected infinitely to a field of homogeneous deformation equal to the average field in the matrix.

The tensors retained in this approximation are those established by Bourgeois?

$C_{M T}=C^{M}\left\{I+\left(\sum_{r=1}^{n} f^{r} Q^{r}\right)\left[I+\sum_{r=1}^{n} f^{r}\left(S^{E s h}-I\right) Q^{r}\right]^{-1}\right\}^{-1}$

With

$$
Q^{r}=\left[\left(C^{M}-C^{r}\right) S^{E s h}-C^{M}\right]^{-1}\left(C^{r}-C^{M}\right)
$$

Where $\mathrm{S}^{\mathrm{Esh}}$ is the tensor of Eshelby and I is the tensor unit of order four.

The expressions of the different components of the Eshelby tensor are listed in several references, including the book of Mura ${ }^{\underline{8}}$.

\subsection{Voigt Model (VT)}

The Voigt approximation, first of all, assumes that the deformation in each phase is identical to the applied macroscopic deformation, so homogeneous ${ }^{\underline{3}}$. This is an approach that leads to a framing of the tensor of effective stress $C_{V T}$ of the composite. We maintain the previous

" $\mathrm{M}$ " and " $\mathrm{r}$ " indexes for matrix and reinforcement.

If the composite is subjected to a deformation of components $E_{i j}$, we will have:

$$
E_{i j}=\frac{1}{V} \int_{V}^{c} \varepsilon_{i j}(x) d v
$$

Where:

$$
V=V^{M}+V^{r}
$$


With $\mathrm{V}\left(\mathrm{m}^{3}\right)$ the volume of the composite, $\mathrm{V}^{\mathrm{M}}$ the volume of the matrix, $\mathrm{V}^{\mathrm{r}}$ the volume of the rienforcement et $e_{i j}(\mathrm{x})$ the microscopic deformation at the point $\mathrm{x}$.

Similarly, from the application of $E_{i j}$ results a stress $\sum_{i j} u$ which is the average volume of the local stress $\sigma_{i j}(x)$ in the material:

$$
\sum_{i j} u=\frac{1}{V} \int_{V}^{r} \sigma_{i j}(x) d v
$$

The generalized Hooke law gives:

$$
\sum_{i j} u=c_{i j k l} \varepsilon_{k l}
$$

With $C_{i j k l}$ the homogenized coefficients of the modulus of elasticity which remains to be determined.

By applying the law of mixtures to (16) and (18), it follows that:

$$
\begin{aligned}
& E_{i j}=\frac{V^{r}}{V} \varepsilon_{i j}^{r}+\frac{V^{M}}{V} \varepsilon_{i j}^{M} \\
& \sum_{i j} u=\frac{V^{r}}{V} \sigma_{i j}^{r}+\frac{V^{M}}{V} \sigma_{i j}^{M}
\end{aligned}
$$

By defining the volume fraction of the reinforcement in the composite by the ratio:

$$
f^{r}=\frac{V^{r}}{V}
$$

Equations (20) and (21) become:

$$
E_{i j}=f^{r} \varepsilon_{i j}^{r}+\left(1-f^{r}\right) \varepsilon_{i j}^{M}
$$

$$
\sum_{i j} y=f^{r} \sigma_{i j}^{r}+\left(1-f^{r}\right) \sigma_{i j}^{M}
$$

The tensor $A^{r}$ of components $A_{i j k l}^{r} \wedge$ defined in Equation (12) allows writing that:

$$
\varepsilon_{i j}^{r}=A_{i j k l}^{r} E_{k l}
$$

The law of behavior at the local level is:

$$
\sigma_{i j}^{r}=C_{i j k l}^{r} \varepsilon_{k l}^{r}
$$

Equations (25) and (26) give:

$$
\sigma_{i j}^{r}=C_{i j k l}^{r} A_{k l m n}^{r} E_{m n}
$$

The constitutive law of the matrix is:

$$
\sigma_{i j}^{M}=C_{i j k l}^{M} \varepsilon_{k l}^{M}
$$

Taking into account Equations (23) and (25), we obtain:

$$
\varepsilon_{i j}^{M}=\frac{1}{\left(1-f^{r}\right)}\left(E_{i j}-f^{r} A_{i j m n}^{r} E_{m n}\right)
$$

From Equations (28) and (29), it follows that:

$$
\sigma_{i j}^{M}=\frac{1}{\left(1-f^{r}\right)} C_{i j k l}^{M}\left(I-f^{r} A_{i j m n}^{r}\right) E_{m n}
$$

With I the tensor unit of order four.

We therefore note that from Equations (24), (27) and (30) and after simplification, we arrive at: 


$$
\sum_{i j} u=\left[C_{i j m n}^{M}+f^{r}\left(C_{i j k l}^{r}-C_{i j k l}^{M}\right) A_{k l m n}^{r}\right] E_{m n}
$$

By identifying Equations (19) and (23), we find the expression of $C_{i j k l}$ :

$$
C_{i j k l}=C_{i j m n}^{M}+f^{r}\left(C_{i j k l}^{r}-C_{i j k l}^{M}\right) A_{k l m n}^{r}
$$

We obtain the model of Voigt with the hypothesis:

$$
\left[A_{i j k l}^{r}\right]=1
$$

In Equation (32):

$$
C_{i j k l}^{V T}=C_{i j m n}^{M}+f^{r}\left(C_{i j k l}^{r}-C_{i j k l}^{M}\right)
$$

or

$$
c_{V T}=c^{M}+f^{r}\left(C^{r}-C^{M}\right)
$$

\section{Simulations}

For simulations, the elastic behaviors of the two phases are determined from the established constitutive law (11). The Young modules obtained are then introduced into each model (14) and (15) and (35) as appropriate to approximate the effective modulus of the composite. This allows us to predict the elastic behavior of the material. This prediction will be done separately according to the temperature and the volume fraction of fibers. During the work of elaboration and characterization ${ }^{1}$, we determined the properties of plasticized starch (without reinforcement) and fibers necessary for simulation. The following table summarizes the properties of the matrix and the fibers.

The factor of security is taken equal to: $C_{s}=2 / 3$ and for the rest, we suppose $f^{r}=f$.

\section{Results and Discussion}

\subsection{Results}

We chose to present the results in the form of graphs to show the evolution of mechanical behavior as a function of temperature and volume fraction. The values of the properties of the different phases summarized in the Table 1 are used in the different models to plot the graphs. Before evaluating the mechanical characteristics of the composite, we will give a representation of each phase using the established mechanical behavior model (11). The application of the model gave us the Figure 1 for the

Table 1. Matrix and fibers properties

\begin{tabular}{|c|c|c|}
\hline Properties & $\begin{array}{c}\text { Plasticized starch (without } \\
\text { reinforcement) }\end{array}$ & Coconut fibers \\
\hline Modulus of elasticity $\left(25^{\circ} \mathrm{C}\right), \mathrm{E}(\mathrm{GPa})$ & 0.037 & 4,500 \\
\hline Resistance to the limit of elasticity, (GPa) & 0.0021 & 0.175 \\
\hline Coefficient of thermal expansion, $\left(^{\circ}\right)$ & $137 \times 10^{-6}$ & $4.7 \times 10^{-6}$ \\
\hline Poisson coefficient & 0.51 & \\
\hline
\end{tabular}




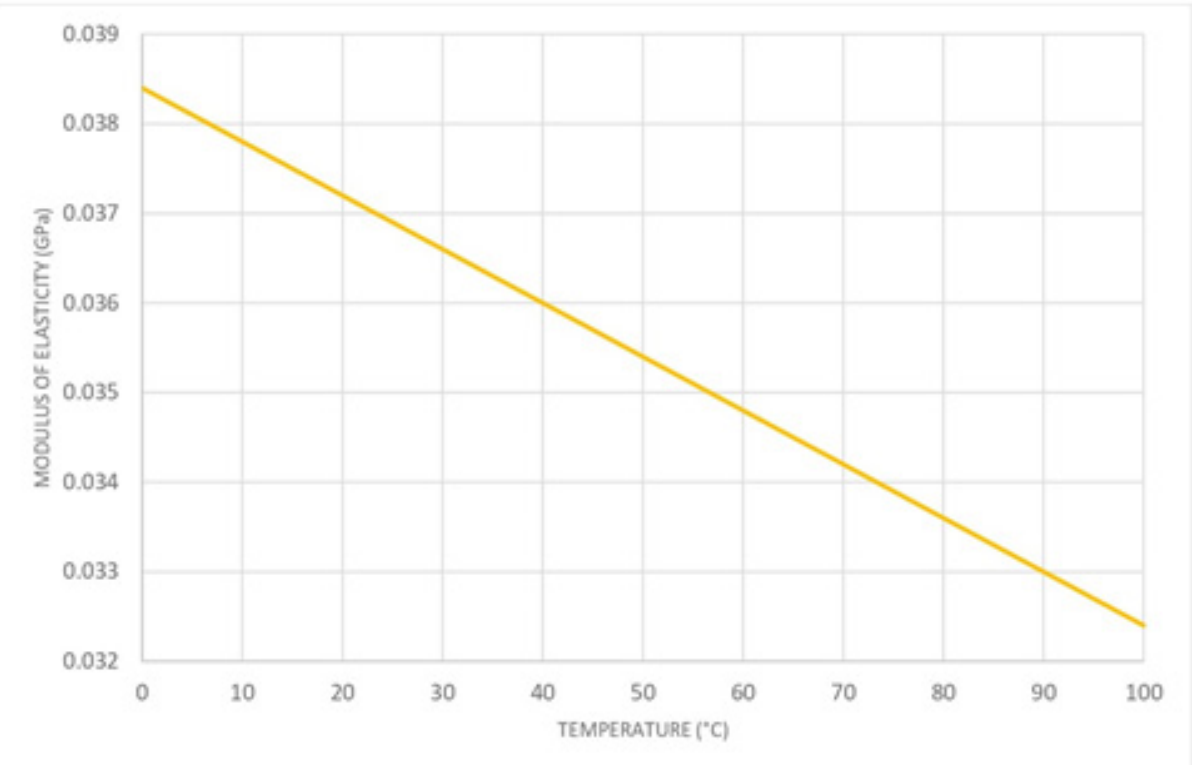

Figure 1. Variation of modulus of elasticity of plasticized starch as a function of temperature.

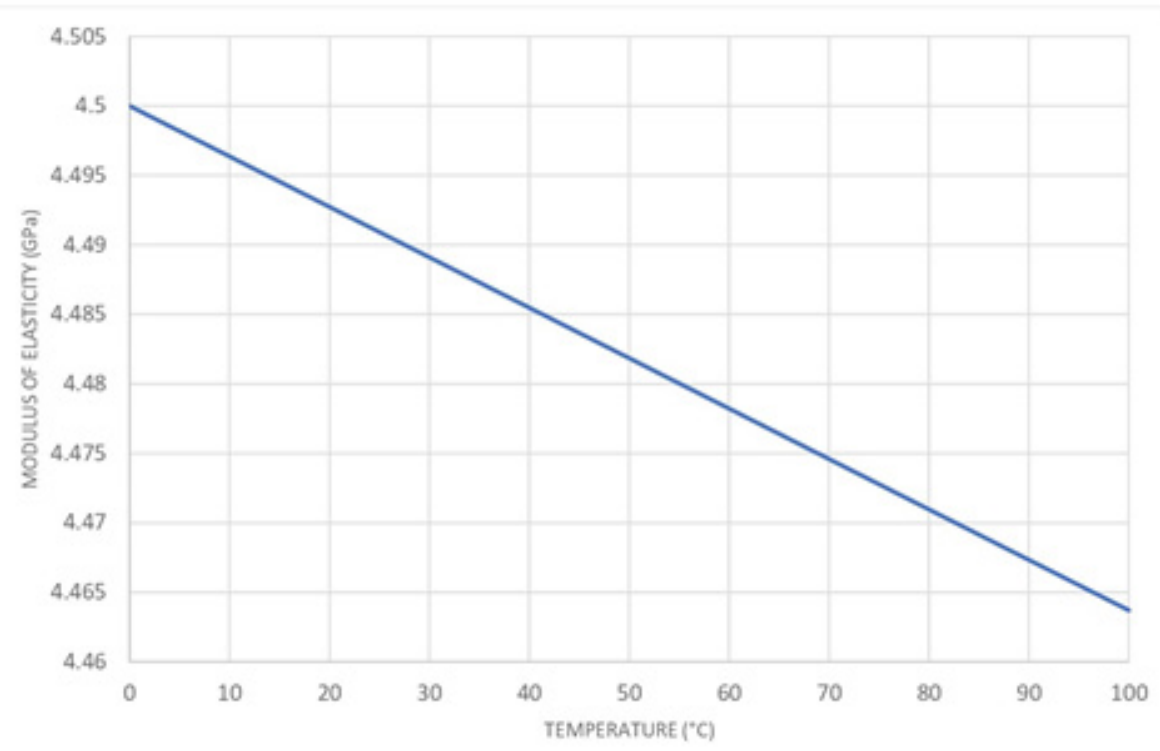

Figure 2. Variation of modulus of elasticity of coconut fibers as a function of temperature.

plasticized starch without reinforcement (matrix) and the Figure 2 (for the coconut fibers (reinforcements)).
Then, we use the Mori-Tanaka and Voigt models to evaluate the Young's modulus of the composite (matrix 


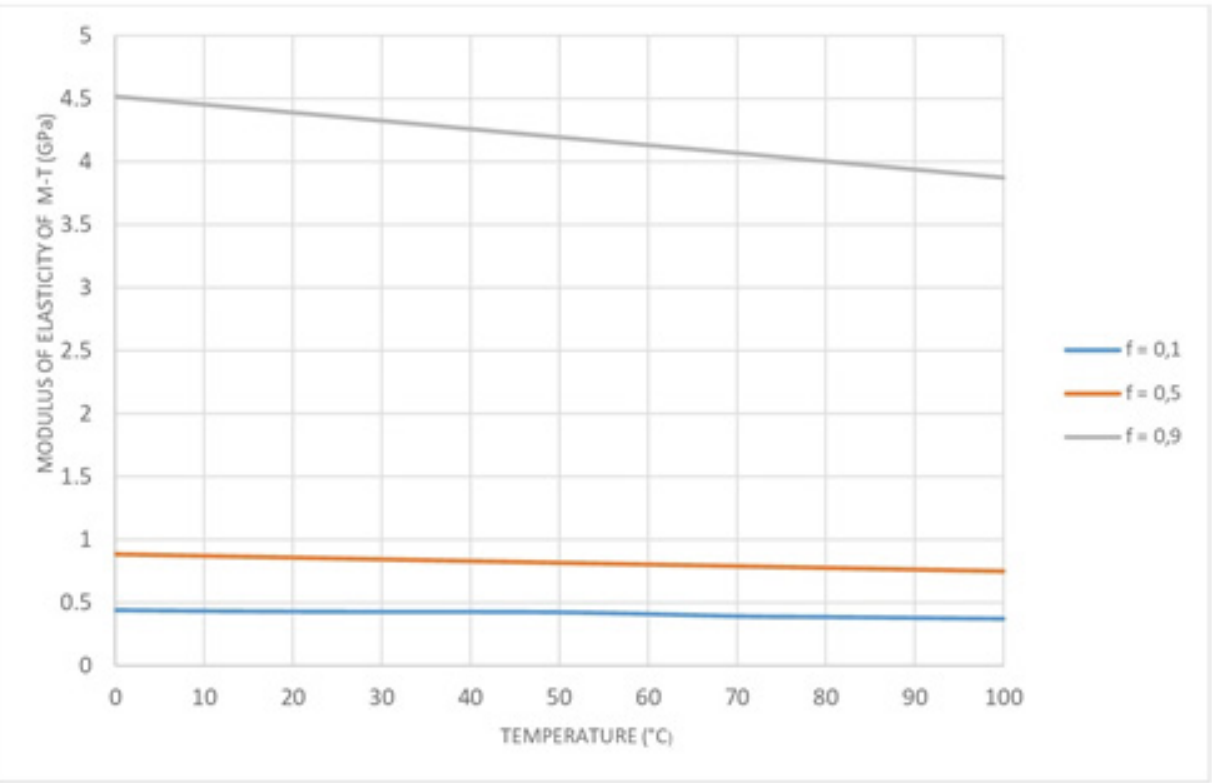

Figure 3. Variation of Mori-Tanaka modulus of elasticity of the composite as a function of temperature.

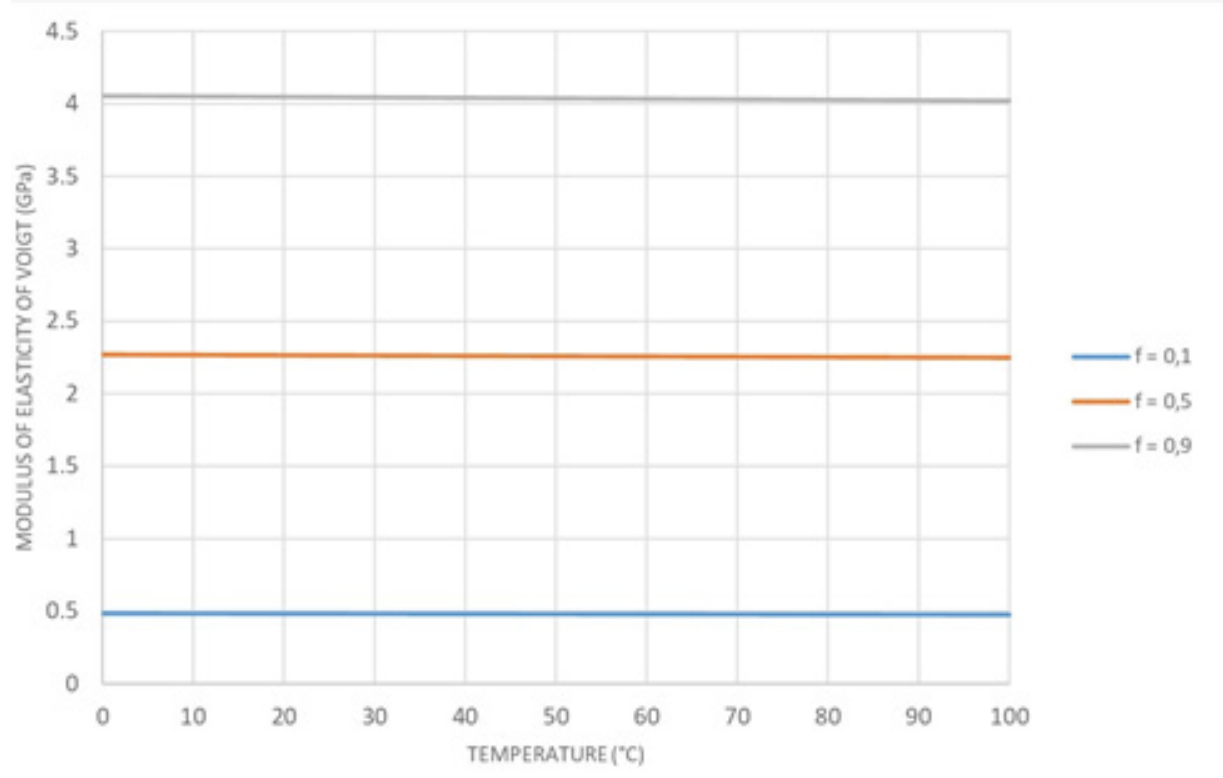

Figure 4. Variation of Voigt modulus of elasticity of the composite as a function of temperature.

+ reinforcements) as a function of temperature at fixed volume fractions. This is illustrated by the Figure 3 .
This makes it possible to predict the elastic behavior as a function of the temperature Figure 4. For both mod- 


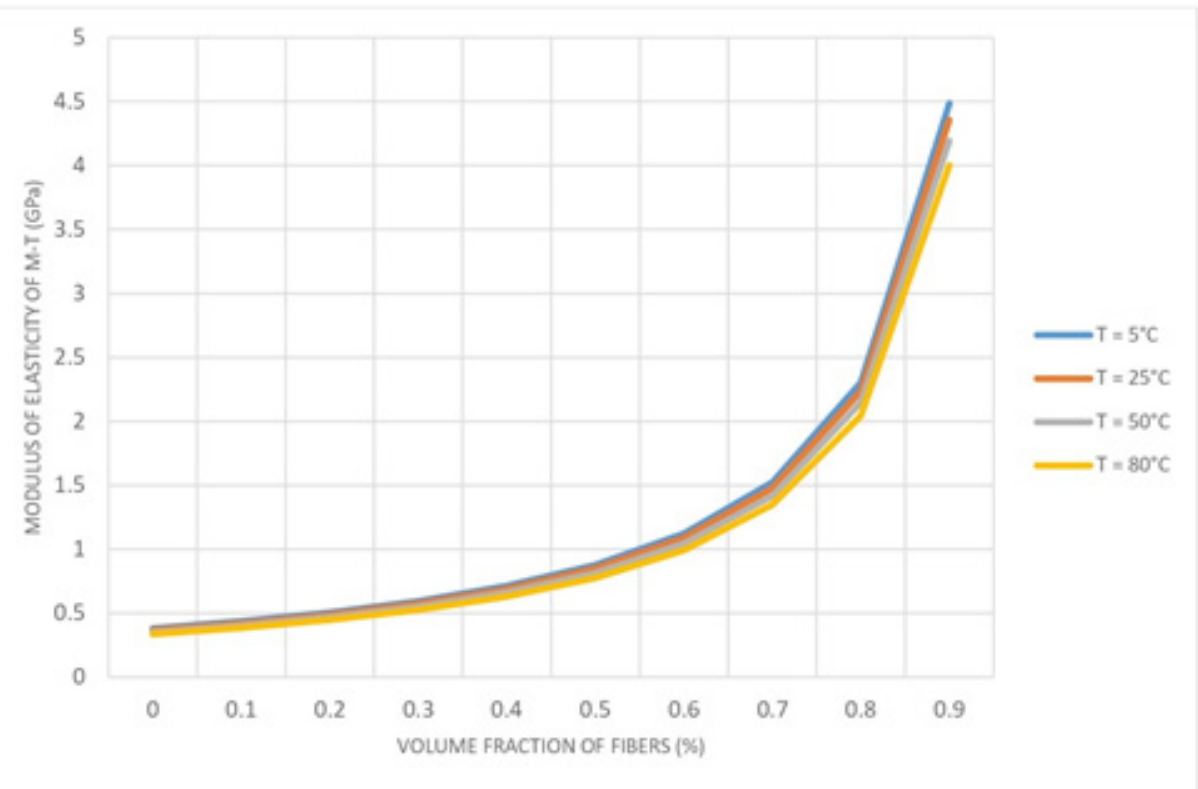

Figure 5. Variation of Mori-Tanaka modulus of elasticity of the composite as a function of volume fraction.

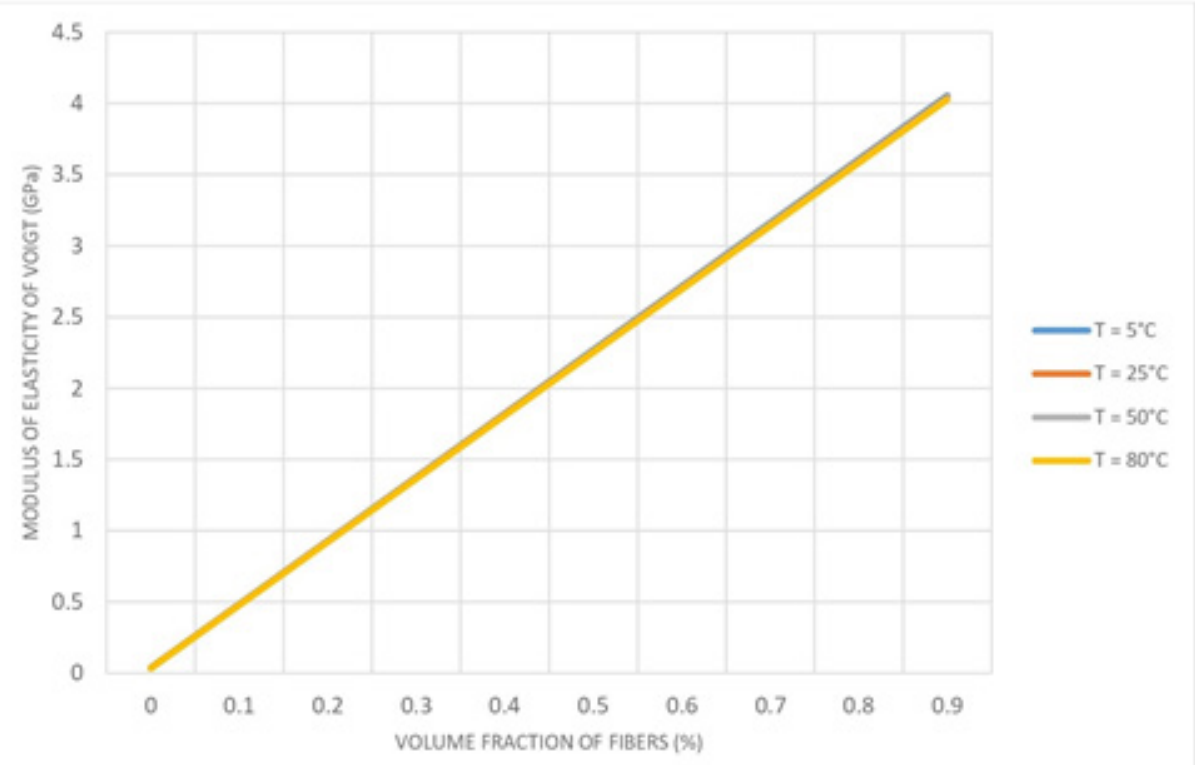

Figure 6. Variation of Voigt modulus of elasticity of the composite as a function of volume fraction.

els, we also determined the modulus of elasticity of the composite as a function of the volume fraction for given temperature values. The Figure 5 and the Figure 6 show, in this case, the evolution of the mechanical behav- 
ior of the composite. This makes it possible to evaluate the mechanical behavior as a function of the volume fraction.

\subsection{Discussion}

All figures show that the modulus of elasticity decreases with increasing temperature. This is consistent with findings found in the work of otherauthors $\frac{4-10}{}$. They specify that the Young's modulus of a material decreases with temperature. Indeed, any input of energy of thermal origin causes the agitation of the atoms causing their distance from each other: The interstitial forces diminish. This means that a rise in temperature leads to a decrease in the mechanical rigidity of the material. The consequence is the decline of Young's modulus $\frac{1-11}{}$. In addition, it can be seen that this variation as a function of temperature is linear in the zone of elastic behavior. This is consistent with the results and $\mathrm{Al}^{12}$. This showed that the variation of the modulus of elasticity is generally linear in the elastic zone of the material.

The graphs also show that the elastic behavior changes with the volume fraction. Our previous study ${ }^{1}$ in which, we have shown that the fibers supply improves the mechanical behavior of the composite confirms this. However, while the Young's modulus of Voigt varies linearly, that of Mori-Tanaka evolves in a parabolic way. This is because the Mori-Tanaka model is polynomial of degree 2 in $\mathrm{f}$ (volume fraction). So we can say that as the amount of fiber increases, the mechanical behavior of the composite improves. For example, for the temperature of $25^{\circ} \mathrm{C}$, the modulus of elasticity in GPA is approximated to $0.42,0.85$ and 4.35 respectively for the volume fractions of $0.1,0.5$ and 0.9 .

\section{Conclusion}

In this study, from a model, we determined the elastic behavior of a material as a function of temperature by evaluating the Young's modulus of reinforcements, matrix and composite. For all materials (fibers, plasticized starch without reinforcements, the composite) it was found that the modulus of elasticity decreases with tempera- ture. This allows us to say that any rise in temperature leads to degradation of the mechanical properties of the material. The modulus of elasticity also increases with the volume fraction. It can be seen that its value tends towards a limit equal to that of the fibers. We can remember that the contribution of fibers improves the behavior of the material with the resistance to the mechanical load, whereas any elevation of the temperature degrades the properties.

\section{References}

1. Doumbia A, Dable PJM, Assanvo EF. Elaboration et caracterisation d'un materiau comosite biodegradable a base d'amidon de manioc renforce par les fibres de mesocarpes de coco. Afrique. Science. 2018; 14(5): $400-14$.

2. Mori T, Tanaka K. Average stress in matrix and average elastic energy of materials with misfitting inclusions. Acta Metallurgica. 1973; 21(5):571-4. https://doi. org/10.1016/0001-6160(73)90064-3.

3. Voigt W. Ueber die Beziehung zwischen den beiden Elasticitatsconstanten isotroper Korper. Wiley Online Library. 1889; 274(12):573-87. https://doi.org/10.1002/ andp. 18892741206.

4. Des Matériaux. 2000. http://www.groupes.polymtl.ca/materiaux/livre.php.

5. Dour G, Durand F, Brechet Y. Relaxation of thermal stresses by dislocation flow and multiplication in the continuous casting of silicon. Materials Science and Engineering. 1997; 5(3):275-88.

6. Ganghoffer JF. Tenseurs, variations et milieux continus, Formulations locale et variationnelle de la mecanique des milieux continus elastiques. Technosup; 2003. p. 184-211.

7. Bourgeois N. Caracterisation et modelisation micromecanique du comportement et de l'endommagement d'un composite a matrice metallique $\mathrm{Al} / \mathrm{SiCp}$. These de l'Ecole Centrale de Paris. 1994; 1:164-79.

8. Mura T. Micromechanics of defects in solids. Springer Netherlands. 1987; 3:14-588.

9. Aide-memoire science des materiaux. 2004. https://blogcaworrioka.firebaseapp.com/210074559X.pdf.

10. Namiko Y, Guzman RV, Wardle BL. Electrical and thermal property enhancement of fiber-reinforced polymer laminate composites through controlled implementation of multi-walled carbon nanotubes. Composites Science and 
Technology. 2012; 72:2009-15. https://doi.org/10.1016/j. compscitech.2012.09.006.

11. Gutierrez J, Le Lay F. Tenue au feu des composite signifuges. Face au risque. 1997; 338:16-24.
12. Jiang Z, Liu X, Zhang H, Li G. An analytical model for elastic stress field distribution in fibre composite with partially debonded interface. Composites Science and Technology. 2005; 65(7):1176-94. https://doi.org/10.1016/j.compscitech.2004.11.014. 